\title{
Some consequences of an existence result by Kiguradze and Partsvania for singular Dirichlet problems
}

\author{
Rubén Figueroa ${ }^{1 *}$ and Rodrigo López Pouso ${ }^{2}$
}

\author{
"Correspondence: \\ ruben.figueroa@usc.es \\ 'Department of Specific Didactics, \\ University of Burgos, Burgos, 09001, \\ Spain \\ Full list of author information is \\ available at the end of the article
}

\begin{abstract}
A sharp theorem by Kiguradze and Partsvania ensures the existence of extremal solutions between given lower and upper solutions for singular Dirichlet problems. This paper has a twofold purpose: first, we present a new sufficient condition for one of Kiguradze and Partsvania's assumptions, and we illustrate its applicability in the study of a new family of examples; second, we combine Kiguradze and Partsvania's theorem with Heikkilä's iterative technique to obtain a new result on the existence of extremal solutions for a more general class of discontinuous and singular functional boundary value problems. In particular, our framework includes classical equations with delay (or advance), singularities with respect to the independent variable, and implicit functional boundary conditions.
\end{abstract}

MSC: $34 \mathrm{~A} 12 ; 34 \mathrm{~A} 36$

Keywords: discontinuous differential equations; singular differential equations; functional differential equations; boundary value problems; equations with delay; equations with advance

\section{Introduction and first results}

We are going to review the results in a paper by Kiguradze and Partsvania [1] and then we are going to use them in the proof of a new more general existence result of extremal solutions for functional and singular second-order problems.

Our revision of the results in [1] is not merely a reproduction, as it includes some contributions of our own. Specifically, Proposition 1.1 provides us with a new sufficient condition for a technical assumption in [1], and we will use it in the analysis of a new family of examples.

Let us start by recalling the main results in [1]. Consider the Dirichlet problem

$$
\begin{aligned}
& u^{\prime \prime}(t)=f\left(t, u(t), u^{\prime}(t)\right), \quad t \in(a, b), \\
& u(a)=c_{1}, \quad u(b)=c_{2},
\end{aligned}
$$

where $-\infty<a<b<+\infty, c_{i} \in \mathbb{R}(i=1,2)$, and $f:(a, b) \times \mathbb{R}^{2} \rightarrow \mathbb{R}$ satisfies

(i) for all $(u, v) \in \mathbb{R}^{2}$ the mapping $t \in(a, b) \mapsto f(t, u, v)$ is measurable;

(ii) for almost all (a.a.) $t \in(a, b)$ the mapping $(u, v) \in \mathbb{R}^{2} \mapsto f(t, u, v)$ is continuous; 
(iii) for every $r>0$ the function defined almost everywhere by

$$
f_{r}^{*}(t)=\max \{|f(t, u, v)|:|u|+|v| \leq r\}
$$

is integrable on $[a+\varepsilon, b-\varepsilon]$ for every sufficiently small $\varepsilon>0$.

Let $A C_{\text {loc }}(a, b)$ denote the set of all real valued functions which are absolutely continuous over each compact subinterval of $(a, b)$.

Definition 1.1 (Kiguradze [2, 3]) A lower (upper) solution of (1.1) is a function $\sigma:(a, b) \rightarrow$ $\mathbb{R}$ such that

(a) $\sigma \in A C_{\mathrm{loc}}(a, b)$ and $\sigma^{\prime}$ admits the representation

$$
\sigma^{\prime}(t)=\gamma(t)+\gamma_{0}(t)
$$

where $\gamma \in A C_{\text {loc }}(a, b)$ and $\gamma_{0}:(a, b) \rightarrow \mathbb{R}$ is a nondecreasing (nonincreasing) function whose first derivative is equal to zero almost everywhere on $(a, b)$;

(b) the inequality

$$
f\left(t, \sigma(t), \sigma^{\prime}(t)\right) \leq \sigma^{\prime \prime}(t) \quad\left(f\left(t, \sigma(t), \sigma^{\prime}(t)\right) \geq \sigma^{\prime \prime}(t)\right)
$$

holds almost everywhere on $(a, b)$;

(c) the limits $\sigma\left(a^{+}\right)$and $\sigma\left(b^{-}\right)$exist and

$$
\sigma\left(a^{+}\right) \leq c_{1}, \quad \sigma\left(b^{-}\right) \leq c_{2} \quad\left(\sigma\left(a^{+}\right) \geq c_{1}, \sigma\left(b^{-}\right) \geq c_{2}\right) .
$$

The previous definitions are very general and allow downwards (upwards) corners in the graphs of the lower (upper) solutions. Readers are referred to [4-6] for similar definitions.

We say that $u:(a, b) \rightarrow \mathbb{R}$ is a solution of (1.1) if it is both a lower and an upper solution, and we are interested in solutions of (1.1) which are extremal in the following sense.

Definition 1.2 Let $Y \subset A C_{\mathrm{loc}}(a, b)$.

We say that a solution $\hat{u}$ of (1.1) is the greatest (smallest) solution of (1.1) in $Y$ if $\hat{u} \in Y$ and $\hat{u} \geq u(\hat{u} \leq u)$ on $(a, b)$ for any other solution $u \in Y$.

When both the smallest and the greatest solutions of (1.1) in $Y$ exist we call them the extremal solutions of (1.1) in $Y$.

The main contribution in [1] concerns the existence of extremal solutions of (1.1) in the presence of lower and upper solutions $\sigma_{1}$ and $\sigma_{2}$. Kiguradze and Partsvania proved their existence result for nonlinearities which belong to the class $B\left(\sigma_{1}, \sigma_{2}\right)$ defined as follows: a function $f:(a, b) \times \mathbb{R}^{2} \rightarrow \mathbb{R}$ belongs to $B\left(\sigma_{1}, \sigma_{2}\right)$ if there exist two constants, $a_{0} \in(a, b)$, $b_{0} \in\left(a_{0}, b\right)$ and a function $\rho \in L^{1}([a, b],[0,+\infty))$ such that $\rho$ is continuous in $(a, b)$ and for any $t_{1} \in\left(a, a_{0}\right), t_{2} \in\left(b_{0}, b\right)$ and continuous $\eta:(a, b) \rightarrow[0,1]$, an arbitrary solution $u$ : $\left(t_{1}, t_{2}\right) \rightarrow \mathbb{R}$ of the differential equation

$$
u^{\prime \prime}(t)=\eta(t) f\left(t, u(t), u^{\prime}(t)\right)
$$


such that $\sigma_{1}(t) \leq u(t) \leq \sigma_{2}(t)$ for $t_{1} \leq t \leq t_{2}$ admits the estimate

$$
\left|u^{\prime}(t)\right| \leq \rho(t) \quad \text { for } t_{1} \leq t \leq t_{2} .
$$

The main result in [1] reads as follows.

Theorem 1.1 [1, Theorem 1] Let $f:(a, b) \times \mathbb{R}^{2} \rightarrow \mathbb{R}$ be a function satisfying (i), (ii), and (iii) and assume that $\sigma_{1}$ and $\sigma_{2}$ are lower and upper solutions of $(1.1)$ such that $\sigma_{1}(t) \leq \sigma_{2}(t)$ for all $t \in(a, b)$. If $f \in B\left(\sigma_{1}, \sigma_{2}\right)$, then (1.1) has extremal solutions in the set

$$
\left[\sigma_{1}, \sigma_{2}\right]=\left\{\xi \in A C_{\mathrm{loc}}(a, b): \sigma_{1}(t) \leq \xi(t) \leq \sigma_{2}(t) \text { for all } t \in(a, b)\right\}
$$

Notice that the condition $f \in B\left(\sigma_{1}, \sigma_{2}\right)$ implies, moreover, that the solutions provided by the previous theorem satisfy the estimate

$$
\left|u^{\prime}(t)\right| \leq \rho(t) \quad \text { for all } t \in(a, b) .
$$

The condition ' $f \in B\left(\sigma_{1}, \sigma_{2}\right)$ ' is hard to check in practice. For that reason, the authors included some sufficient hypotheses which imply it. One of them is a one-sided Nagumo condition, in the spirit of [3]. The following is a slight modification of Corollary $1_{1}$ in [1].

Corollary 1.1 Assume that there exist $\sigma_{1}, \sigma_{2}$ which are, respectively, a lower and an upper solution of problem (1.1) such that $\sigma_{1}(t) \leq \sigma_{2}(t)$ for all $t \in(a, b)$ and that the following conditions hold:

$\left(\mathrm{H}_{1}\right)$ For each $u \in\left[\inf _{t \in(a, b)} \sigma_{1}(t), \sup _{t \in(a, b)} \sigma_{2}(t)\right]$ and all $v \in \mathbb{R}$ the mapping $t \in(a, b) \mapsto$ $f(t, u, v)$ is measurable.

$\left(\mathrm{H}_{2}\right)$ For almost all $t \in(a, b)$, all $u \in\left[\sigma_{1}(t), \sigma_{2}(t)\right]$ and all $v \in \mathbb{R}$ the functions $f(t, \cdot, v)$ and $f(t, u, \cdot)$ are continuous.

$\left(\mathrm{H}_{3}\right)$ For every $r>0$ the function

$$
f_{r}^{*}(t)=\max \{|f(t, u, v)|:|u|+|v| \leq r\}
$$

is integrable on $[a+\varepsilon, b-\varepsilon]$ for every sufficiently small $\varepsilon>0$.

$\left(\mathrm{H}_{4}\right)$ There exist $a_{0} \in(a, b), b_{0} \in\left(a_{0}, b\right), \psi \in L_{\mathrm{loc}}^{1}((a, b),[0,+\infty))$, and $\varphi \in \mathcal{C}(\mathbb{R},(0,+\infty))$ such that for a.a. $t \in\left(a, b_{0}\right)$, all $u \in\left[\sigma_{1}(t), \sigma_{2}(t)\right]$ and all $v \in \mathbb{R}$ we have

$$
f(t, u, v) \operatorname{sgn} v \geq-\psi(t) \varphi(v)
$$

and for a.a. $t \in\left(a_{0}, b\right)$, all $u \in\left[\sigma_{1}(t), \sigma_{2}(t)\right]$ and all $v \in \mathbb{R}$ we have

$$
f(t, u, v) \operatorname{sgn} v \leq \psi(t) \varphi(v) .
$$

Moreover, for

$$
r=\max \left\{\frac{\sigma_{2}\left(a_{0}\right)-\sigma_{1}\left(b_{0}\right)}{b_{0}-a_{0}}, \frac{\sigma_{2}\left(b_{0}\right)-\sigma_{1}\left(a_{0}\right)}{b_{0}-a_{0}}\right\},
$$


there exists a continuous function $\rho:(a, b) \rightarrow[r,+\infty)$ such that $\rho \in L^{1}(a, b)$ and for all $t \in(a, b)$ we have

$$
\min \left\{\int_{-\rho(t)}^{-r} \frac{d v}{\varphi(v)}, \int_{r}^{\rho(t)} \frac{d v}{\varphi(v)}\right\}>\max \left\{\int_{a_{0}}^{t} \psi(s) d s, \int_{t}^{b_{0}} \psi(s) d s\right\}
$$

Then problem (1.1) has the extremal solutions in $\left[\sigma_{1}, \sigma_{2}\right]$. Moreover, if $u$ is a solution of (1.1) such that $\sigma_{1} \leq u \leq \sigma_{2}$, then $\left|u^{\prime}(t)\right| \leq \rho(t)$ for all $t \in(a, b)$.

Proof We only have to show that $f \in B\left(\sigma_{1}, \sigma_{2}\right)$ and then the conclusion follows from Theorem 1.1. To do this, let $t_{1}, t_{2}$ be such that $a<t_{1}<a_{0}<b_{0}<t_{2}<b, \eta \in \mathcal{C}((a, b),[0,1])$, and $u \in\left[\sigma_{1}, \sigma_{2}\right]$ is an arbitrary solution of the differential equation

$$
u^{\prime \prime}(t)=\eta(t) f\left(t, u(t), u^{\prime}(t)\right) \quad \text { for a.a. } t \in\left[t_{1}, t_{2}\right] .
$$

We claim that $\left|u^{\prime}(t)\right| \leq \rho(t)$ for all $t \in\left[t_{1}, t_{2}\right]$. Indeed, as

$$
-r \leq \frac{\sigma_{1}\left(b_{0}\right)-\sigma_{2}\left(a_{0}\right)}{b_{0}-a_{0}} \leq \frac{u\left(b_{0}\right)-u\left(a_{0}\right)}{b_{0}-a_{0}} \leq \frac{\sigma_{2}\left(b_{0}\right)-\sigma_{1}\left(a_{0}\right)}{b_{0}-a_{0}} \leq r,
$$

there exists $\tau_{0} \in\left[a_{0}, b_{0}\right]$ such that $u^{\prime}\left(\tau_{0}\right) \leq r$.

Reasoning by contradiction, assume that for some $\tau_{1} \in\left[t_{1}, t_{2}\right]$ we have $u^{\prime}\left(\tau_{1}\right)>\rho\left(\tau_{1}\right)$ and, without loss of generality, that $u^{\prime}(t) \geq 0$ for all $t \in\left[\tau_{0}, \tau_{1}\right]$ or $\left[\tau_{1}, \tau_{0}\right]$. Then

$$
\int_{r}^{\rho\left(\tau_{1}\right)} \frac{d v}{\varphi(v)} \leq \int_{u^{\prime}\left(\tau_{0}\right)}^{u^{\prime}\left(\tau_{1}\right)} \frac{d v}{\varphi(v)}=\int_{\tau_{0}}^{\tau_{1}} \frac{u^{\prime \prime}(s) d s}{\varphi\left(u^{\prime}(s)\right)}=\int_{\tau_{0}}^{\tau_{1}} \frac{\eta(s) f\left(s, u(s), u^{\prime}(s)\right) d s}{\varphi\left(u^{\prime}(s)\right)}
$$

so, in case $\tau_{0}<\tau_{1}$, we have

$$
\int_{r}^{\rho\left(\tau_{1}\right)} \frac{d v}{\varphi(v)} \leq \int_{\tau_{0}}^{\tau_{1}} \eta(s) \psi(s) d s \leq \int_{\tau_{0}}^{\tau_{1}} \psi(s) d s \leq \int_{a_{0}}^{\tau_{1}} \psi(s) d s,
$$

and if $\tau_{1}<\tau_{0}$, then

$$
\int_{r}^{\rho\left(\tau_{1}\right)} \frac{d \nu}{\varphi(v)} \leq \int_{\tau_{1}}^{\tau_{0}} \eta(s) \psi(s) d s \leq \int_{\tau_{1}}^{\tau_{0}} \psi(s) d s \leq \int_{\tau_{1}}^{b_{0}} \psi(s) d s .
$$

The previous two inequalities contradict (1.5), thus proving that $u^{\prime}\left(\tau_{1}\right)>\rho\left(\tau_{1}\right)$ is possible for no $\tau_{1} \in\left[t_{1}, t_{2}\right]$.

One can prove in an analogous way that $u^{\prime}(t) \geq-\rho(t)$ for all $t \in\left[t_{1}, t_{2}\right]$.

Finding a function $\rho(t)$ to check (1.5) with is not easy, but the following proposition quite simplifies that task.

Proposition 1.1 Let $\psi \in L_{\mathrm{loc}}^{1}((a, b),[0,+\infty)), \varphi \in \mathcal{C}(\mathbb{R},(0,+\infty)), a_{0} \in(a, b), b_{0} \in\left(a_{0}, b\right)$, and $r>0$.

Define

$$
\phi_{1}(x)=\int_{r}^{x} \frac{d v}{\varphi(v)} \quad \text { for } x \geq r
$$


and

$$
\phi_{2}(x)=\int_{x}^{-r} \frac{d v}{\varphi(v)} \quad \text { for } x \leq-r
$$

If the functions

$$
\begin{aligned}
& \rho_{1}(t)=\phi_{1}^{-1}\left(\max \left\{\int_{a_{0}}^{t} \psi(s) d s, \int_{t}^{b_{0}} \psi(s) d s\right\}+r\right), \quad t \in(a, b), \\
& \rho_{2}(t)=-\phi_{2}^{-1}\left(\max \left\{\int_{a_{0}}^{t} \psi(s) d s, \int_{t}^{b_{0}} \psi(s) d s\right\}+r\right), \quad t \in(a, b),
\end{aligned}
$$

are well defined and integrable in $(a, b)$, then condition (1.5) holds with

$$
\rho(t)=\max \left\{\rho_{1}(t), \rho_{2}(t)\right\}, \quad t \in(a, b) .
$$

Proof The assumptions guarantee that $\rho:(a, b) \rightarrow[r,+\infty)$ is continuous and integrable.

Now let $t \in(a, b)$ be fixed. If $\rho(t)=\rho_{1}(t)$, then

$$
\begin{aligned}
\int_{r}^{\rho(t)} \frac{d v}{\varphi(v)} & =\int_{r}^{\rho_{1}(t)} \frac{d v}{\varphi(v)}=\phi_{1}\left(\rho_{1}(t)\right) \\
& =\max \left\{\int_{a_{0}}^{t} \psi(s) d s, \int_{t}^{b_{0}} \psi(s) d s\right\}+r \\
& >\max \left\{\int_{a_{0}}^{t} \psi(s) d s, \int_{t}^{b_{0}} \psi(s) d s\right\} .
\end{aligned}
$$

On the other hand, the identity $\rho(t)=\rho_{1}(t)$ yields $\rho_{1}(t) \geq \rho_{2}(t)$; hence

$$
\int_{-\rho(t)}^{-r} \frac{d v}{\varphi(v)}=\int_{-\rho_{1}(t)}^{-r} \frac{d v}{\varphi(v)} \geq \phi_{2}\left(-\rho_{2}(t)\right)>\max \left\{\int_{a_{0}}^{t} \psi(s) d s, \int_{t}^{b_{0}} \psi(s) d s\right\} .
$$

The proof is similar if $\rho(t)=\rho_{2}(t)$.

We close this section with a new example of the applicability of Kiguradze and Partsvania's results. Notice how our Proposition 1.1 simplifies the verification of condition $\left(\mathrm{H}_{4}\right)$ in Corollary 1.1 .

Example 1.1 For every $n \in \mathbb{N}$ and every $\lambda \in(1,+\infty)$ the singular Dirichlet problem

$$
\begin{aligned}
& u^{\prime \prime}(t)=f\left(t, u(t), u^{\prime}(t)\right)=\frac{u^{n}(t) u^{\prime}(t)}{\lambda t(1-t)}, \quad t \in(0,1), \\
& u(0)=-1, \quad u(1)=1,
\end{aligned}
$$

has the extremal solutions between the lower solution $\sigma_{1} \equiv-1$ and the upper solution $\sigma_{2} \equiv 1$.

Conditions $\left(\mathrm{H}_{1}\right)$ through $\left(\mathrm{H}_{3}\right)$ in Corollary 1.1 are obviously satisfied. The hardest part concerns showing that $\left(\mathrm{H}_{4}\right)$ is satisfied with, for instance, the following choice of the functions and the constants: let us define $\psi(t)=1 /[t(1-t)]$ for all $t \in(0,1), \varphi(v)=(|v|+1) / \lambda$ for all $v \in \mathbb{R}, a_{0}=1 / 4, b_{0}=1 / 2$, and, according to (1.4), $r=8$. 
First, notice that for all $t \in(0,1)$, all $v \in \mathbb{R}$ and all $u \in\left[\sigma_{1}, \sigma_{2}\right]=[-1,1]$ we have

$$
|f(t, u, v)|=\frac{|u|^{n}|v|}{\lambda t(1-t)} \leq \psi(t) \varphi(v),
$$

and this implies (1.2) and (1.3).

Let us use now Proposition 1.1 to show that (1.5) is satisfied too. First, we have to define a function on $[r,+\infty)=[8,+\infty)$ by

$$
\phi_{1}(x)=\int_{r}^{x} \frac{d v}{\varphi(v)}=\int_{8}^{x} \frac{\lambda}{v+1} d v=\lambda \ln \frac{x+1}{9}
$$

and for all $x \leq-r=-8$ we define

$$
\phi_{2}(x)=\int_{x}^{-r} \frac{d v}{\varphi(v)}=\lambda \ln \frac{1-x}{9}=\phi_{1}(-x) .
$$

Now we have to check that the following (continuous) functions are also integrable on $(0,1)$ :

$$
\begin{aligned}
& \rho_{1}(t)=\phi_{1}^{-1}\left(\max \left\{\int_{1 / 4}^{t} \psi(s) d s, \int_{t}^{1 / 2} \psi(s) d s\right\}+8\right), \quad t \in(0,1) \\
& \rho_{2}(t)=-\phi_{2}^{-1}\left(\max \left\{\int_{1 / 4}^{t} \psi(s) d s, \int_{t}^{1 / 2} \psi(s) d s\right\}+8\right), \quad t \in(0,1) .
\end{aligned}
$$

Notice that the relation between $\phi_{1}$ and $\phi_{2}$ implies that $\rho_{2}(t)=\rho_{1}(t)$ for all $t \in(0,1)$, so our problem reduces to proving that $\rho_{1}$ is integrable.

For all $t \in(0,1 / 4)$ we have

$$
\int_{1 / 4}^{t} \psi(s) d s<0
$$

and therefore

$$
\max \left\{\int_{1 / 4}^{t} \psi(s) d s, \int_{t}^{1 / 2} \psi(s) d s\right\}=\int_{t}^{1 / 2} \psi(s) d s=\ln \frac{1-t}{t} .
$$

Hence,

$$
\rho_{1}(t)=9 e^{8 / \lambda}\left(\frac{1-t}{t}\right)^{1 / \lambda}-1 \quad \text { for all } t \in(0,1 / 4)
$$

which is integrable on $(0,1 / 4)$ because $1 / \lambda \in(0,1)$.

Similar computations show that $\rho_{1}$ is integrable on $(1 / 2,1)$, and then it is integrable on the whole of $(0,1)$ because it is continuous. Hence, Proposition 1.1 ensures that condition (1.5) holds with $\rho=\rho_{1}$.

\section{Singular functional problems}

In this section we are concerned with a generalization of problem (1.1) which includes both past and future dependence. To do so, let us fix $r_{-}, r_{+} \geq 0$, define

$$
\mathcal{Y}=\left\{\gamma:\left[a-r_{-}, b+r_{+}\right] \rightarrow \mathbb{R}: \gamma \text { is continuous and } \gamma_{\mid(a, b)} \in A C_{\mathrm{loc}}(a, b)\right\} \text {, }
$$


and consider the problem

$$
\left\{\begin{array}{l}
u^{\prime \prime}(t)=f\left(t, u(t), u^{\prime}(t), u\right) \text { for a.a. } t \in(a, b), \\
u(t)=B_{-}(t, u(t), u) \text { for all } t \in I_{-}=\left[a-r_{-}, a\right], \\
u(t)=B_{+}(t, u(t), u) \text { for all } t \in I_{+}=\left[b, b+r_{+}\right],
\end{array}\right.
$$

where $f:(a, b) \times \mathbb{R}^{2} \times \mathcal{Y} \rightarrow \mathbb{R}$ and $B_{\mp}: I_{\mp} \times \mathbb{R} \times \mathcal{Y} \rightarrow \mathbb{R}$.

Notice that, in general, solutions of (1.1) are defined on an interval which is greater than $(a, b)$ and, moreover, the differential equation depends upon the behavior of the solution in that extended interval. This implies, in particular, that we can study equations with deviated arguments of the form

$$
u^{\prime \prime}(t)=f(t, u(t), u(\tau(t)))
$$

where $\tau: t \in(a, b) \rightarrow\left[a-r_{-}, b+r_{+}\right]$is measurable. Moreover, classical start-final functions for problems with delay or advance are also included in (2.1): we only have to take

$$
B_{-}(t, u(t), u)=\phi_{-}(t), \quad B_{+}(t, u(t), u)=\phi_{+}(t)
$$

where $\phi_{-}$and $\phi_{+}$represent, respectively, the initial and the final state of the solution. Problem (2.1) also includes multipoint boundary conditions, which have received a lot of attention in the last few years; see $[7,8]$.

We begin by introducing the concepts of lower and upper solutions for problem (2.1). We do this simply by extending Definition 1.1 in the obvious way.

Definition 2.1 We say that $\sigma \in \mathcal{Y}$ is a lower solution (respectively, an upper solution) of problem (2.1) if it satisfies the following conditions:

(a) $\sigma_{\mid(a, b)} \in A C_{\mathrm{loc}}(a, b)$ and $\sigma^{\prime}$ admits the representation

$$
\sigma^{\prime}(t)=\gamma(t)+\gamma_{0}(t)
$$

where $\gamma \in A C_{\mathrm{loc}}(a, b)$ and $\gamma_{0}:(a, b) \rightarrow \mathbb{R}$ is a nondecreasing (respectively, nonincreasing) function whose first derivative is equal to zero almost everywhere on $(a, b)$;

(b) the inequality

$$
f\left(t, \sigma(t), \sigma^{\prime}(t), \sigma\right) \leq \sigma^{\prime \prime}(t) \quad\left(\text { respectively, } f\left(t, \sigma(t), \sigma^{\prime}(t), \sigma\right) \geq \sigma^{\prime \prime}(t)\right)
$$

holds almost everywhere on $(a, b)$;

(c) for all $t \in I_{-}$we have

$$
\sigma(t) \leq B_{-}(t, \sigma(t), \sigma) \quad\left(\text { respectively, } \sigma(t) \geq B_{-}(t, \sigma(t), \sigma)\right),
$$

and for all $t \in I_{+}$we have

$$
\sigma(t) \leq B_{+}(t, \sigma(t), \sigma) \quad\left(\text { respectively, } \sigma(t) \geq B_{+}(t, \sigma(t), \sigma)\right) .
$$


Our existence result for (2.1) will be proven by means of Kiguradze and Partsvania's results described in the previous section along with Heikkiläs generalized iterative technique. The following result will be essential.

Lemma 2.1 [9, Theorem 1.2.2] Let $\mathcal{Y}$ be a subset of an ordered metric space $\mathcal{X},[\alpha, \beta]$ a nonempty order interval in $\mathcal{Y}$, and $G:[\alpha, \beta] \rightarrow[\alpha, \beta]$ a nondecreasing mapping. If $\left\{G u_{n}\right\}_{n=1}^{\infty}$ converges in $\mathcal{Y}$ whenever $\left\{u_{n}\right\}_{n=1}^{\infty}$ is a monotone sequence in $[\alpha, \beta]$, then $G$ has in $[\alpha, \beta]$ the extremal fixed points, $u_{*}$ and $u^{*}$, which satisfy

$$
u_{*}=\min \{u: G u \leq u\}, \quad u^{*}=\max \{u: u \leq G u\} .
$$

We are at last in a position to introduce and prove our main result.

Theorem 2.1 Let $\sigma_{1}$ and $\sigma_{2}$ be, respectively, a lower and an upper solution of problem (2.1) such that $\sigma_{1} \leq \sigma_{2}$ on $\left[a-r_{-}, b+r_{+}\right]$, put

$$
\left[\sigma_{1}, \sigma_{2}\right]=\left\{\gamma \in \mathcal{Y}: \sigma_{1}(t) \leq \gamma(t) \leq \sigma_{2}(t) \text { for all } t \in\left[a-r_{-}, b+r_{+}\right]\right\},
$$

and assume that the following conditions hold:

$\left(\mathrm{F}_{1}\right)$ For each $\gamma \in\left[\sigma_{1}, \sigma_{2}\right]$ the mapping $f_{\gamma}(t, u, v)=f(t, u, v, \gamma)$ satisfies conditions (i), (ii), and (iii), and $f_{\gamma} \in B\left(\sigma_{1}, \sigma_{2}\right)$ uniformly in $\gamma \in\left[\sigma_{1}, \sigma_{2}\right]$, in the sense that the function $\rho$ does not depend on $\gamma$.

$\left(\mathrm{F}_{2}\right)$ For a.a. $t \in(a, b)$, all $u \in\left[\sigma_{1}(t), \sigma_{2}(t)\right]$ and all $v \in \mathbb{R}$ the mapping $f(t, u, v, \cdot)$ is nonincreasing on $\left[\sigma_{1}, \sigma_{2}\right]$.

$\left(\mathrm{B}_{1}\right)$ For each $\varepsilon>0$ there exists $\delta>0$ such that for any $t, s \in I_{\mp}$ the inequality $|t-s|<\delta$ implies

$$
\left|B_{\mp}(t, u, \gamma)-B_{\mp}(s, u, \gamma)\right|<\varepsilon
$$

for all $u \in\left[\min _{I_{\mp}} \sigma_{1}, \max _{I_{\mp}} \sigma_{2}\right]$ and all $\gamma \in\left[\sigma_{1}, \sigma_{2}\right]$.

$\left(\mathrm{B}_{2}\right)$ There exists $\lambda \in[0,1)$ such that for all $t \in I_{\mp}$ and all $\gamma \in\left[\sigma_{1}, \sigma_{2}\right]$ we have

$$
\left|B_{\mp}(t, u, \gamma)-B_{\mp}(t, v, \gamma)\right| \leq \lambda|u-v| \quad\left(u, v \in\left[\min _{I_{\mp}} \sigma_{1}, \max _{I_{\mp}} \sigma_{2}\right]\right) .
$$

$\left(\mathrm{B}_{3}\right)$ For all $t \in I_{\mp}$ and all $u \in\left[\min _{I_{\mp}} \sigma_{1}, \max _{I_{\mp}} \sigma_{2}\right]$, the mapping $B_{\mp}(t, u, \cdot)$ is nondecreasing in $\left[\sigma_{1}, \sigma_{2}\right]$.

Then problem (2.1) has extremal solutions in $\left[\sigma_{1}, \sigma_{2}\right]$.

Proof Consider the set $\mathcal{X}=\left\{\gamma:\left[a-r_{-}, b+r_{+}\right] \rightarrow \mathbb{R}: \gamma\right.$ is continuous $\}$, which becomes an ordered metric space (in fact, a Banach space) when endowed with the supremum norm and the pointwise ordering. In $\mathcal{X}$ consider the subset $\mathcal{Y}$ and the ordered interval $\left[\sigma_{1}, \sigma_{2}\right] \subset \mathcal{Y}$. Now, define an operator $G:\left[\sigma_{1}, \sigma_{2}\right] \rightarrow\left[\sigma_{1}, \sigma_{2}\right]$ as follows: for each $\gamma \in\left[\sigma_{1}, \sigma_{2}\right]$, $G \gamma$ is the greatest solution in $\left[\sigma_{1}, \sigma_{2}\right]$ of the Dirichlet problem

$$
\left(P_{\gamma}\right)\left\{\begin{array}{l}
u^{\prime \prime}(t)=f_{\gamma}\left(t, u(t), u^{\prime}(t)\right)=f\left(t, u(t), u^{\prime}(t), \gamma\right) \text { for a.a. } t \in(a, b), \\
u(t)=\Gamma_{-}(t) \quad \text { for all } t \in\left[a-r_{-}, a\right] \\
u(t)=\Gamma_{+}(t) \quad \text { for all } t \in\left[b, b+r_{+}\right]
\end{array}\right.
$$


where for each $t \in\left[a-r_{-}, a\right]$ (respectively, each $\left.t \in\left[b, b+r_{+}\right]\right), \Gamma_{-}(t)$ (respectively, $\Gamma_{+}(t)$ ) is the unique fixed point $x \in\left[\sigma_{1}(t), \sigma_{2}(t)\right]$ of the contractive mapping $B_{-}(t, \cdot, \gamma)$ (respectively, $\left.B_{+}(t, \cdot, \gamma)\right)$.

Claim 1: Operator $G$ is well defined. Fix $\gamma \in\left[\sigma_{1}, \sigma_{2}\right]$. First, the definitions of lower and upper solutions and condition $\left(\mathrm{B}_{3}\right)$ ensure that for any $t \in[a-r, a]$ we have

$$
\sigma_{1}(t) \leq B_{-}\left(t, \sigma_{1}(t), \sigma_{1}\right) \leq B_{-}\left(t, \sigma_{1}(t), \gamma\right)
$$

and $\sigma_{2}(t) \geq B_{-}\left(t, \sigma_{2}(t), \gamma\right)$. Hence $B_{-}(t, \cdot, \gamma)$ has at least one fixed point in $\left[\sigma_{1}(t), \sigma_{2}(t)\right]$, which is unique by virtue of $\left(\mathrm{B}_{2}\right)$. Hence, the number $\Gamma_{-}(t)$ is well defined for every $t \in I_{-}$; let us prove now that $\Gamma_{-}$is continuous on $I_{-}$. To do so, let us fix $s, t \in I_{-}$and notice that

$$
\begin{aligned}
\left|\Gamma_{-}(t)-\Gamma_{-}(s)\right|= & \left|B_{-}\left(t, \Gamma_{-}(t), \gamma\right)-B_{-}\left(s, \Gamma_{-}(s), \gamma\right)\right| \\
\leq & \left|B_{-}\left(t, \Gamma_{-}(t), \gamma\right)-B_{-}\left(t, \Gamma_{-}(s), \gamma\right)\right| \\
& +\left|B_{-}\left(t, \Gamma_{-}(s), \gamma\right)-B_{-}\left(s, \Gamma_{-}(s), \gamma\right)\right| \\
\leq & \lambda\left|\Gamma_{-}(t)-\Gamma_{-}(s)\right|+\left|B_{-}\left(t, \Gamma_{-}(s), \gamma\right)-B_{-}\left(s, \Gamma_{-}(s), \gamma\right)\right|,
\end{aligned}
$$

hence

$$
\left|\Gamma_{-}(t)-\Gamma_{-}(s)\right| \leq \frac{1}{1-\lambda}\left|B_{-}\left(t, \Gamma_{-}(s), \gamma\right)-B_{-}\left(s, \Gamma_{-}(s), \gamma\right)\right|,
$$

and therefore $\Gamma_{-}$is continuous by virtue of $\left(\mathrm{B}_{1}\right)$.

A similar argument shows that $\Gamma_{+}(t)$ is well defined and continuous too.

Now, condition $\left(\mathrm{F}_{1}\right)$ guarantees by application of Theorem 1.1 that the extremal solutions in $\left[\sigma_{1}, \sigma_{2}\right]$ of problem $\left(P_{\gamma}\right)$ exist and, in particular, $G \gamma$ is well defined.

Moreover, condition $\left(\mathrm{F}_{1}\right)$ also ensures that there exists a continuous and integrable function $\rho:(a, b) \rightarrow \mathbb{R}$ such that $\left|(G \gamma)^{\prime}(t)\right| \leq \rho(t)$ for all $t \in(a, b)$ and all $\gamma \in\left[\sigma_{1}, \sigma_{2}\right]$.

Claim 2: Operator $G$ is nondecreasing. Let $\gamma_{1}, \gamma_{2} \in\left[\sigma_{1}, \sigma_{2}\right]$ be such that $\gamma_{1} \leq \gamma_{2}$. Then by condition $\left(\mathrm{F}_{2}\right)$ we have for a.a. $t \in(a, b)$ that

$$
G \gamma_{1}^{\prime \prime}(t)=f\left(t, G \gamma_{1}(t), G \gamma_{1}^{\prime}(t), \gamma_{1}\right) \geq f\left(t, G \gamma_{1}(t), G \gamma_{1}^{\prime}(t), \gamma_{2}\right) .
$$

Let us prove now that $G \gamma_{1}(t) \leq G \gamma_{2}(t)$ for each $t \in I_{-}$. Indeed, condition $\left(\mathrm{B}_{3}\right)$ yields

$$
G \gamma_{1}(t)=B_{-}\left(t, G \gamma_{1}(t), \gamma_{1}\right) \leq B_{-}\left(t, G \gamma_{1}(t), \gamma_{2}\right),
$$

which implies that $B_{-}\left(t, \cdot, \gamma_{2}\right)$ has its fixed point, namely $G \gamma_{2}(t)$, inside the interval $\left[G \gamma_{1}(t), \sigma_{2}(t)\right]$. Hence, $G \gamma_{1} \leq G \gamma_{2}$ on $I_{-}$.

A similar argument shows that $G \gamma_{1}(t) \leq G \gamma_{2}(t)$ for all $t \in I_{+}$.

Summing up, $G \gamma_{1}$ is a lower solution of problem $\left(P_{\gamma_{2}}\right)$, whose greatest solution between $\sigma_{1}$ and $\sigma_{2}$ is precisely $G \gamma_{2}$. Therefore $G \gamma_{1} \leq G \gamma_{2}$ on the whole of $\left[a-r_{-}, b+r_{+}\right]$.

Claim 3: Operator $G$ has the extremal fixed points. Let $\left\{\gamma_{n}\right\}_{n=1}^{\infty} \subset\left[\sigma_{1}, \sigma_{2}\right]$ be a monotone sequence. As $G$ is nondecreasing, the sequence $\left\{z_{n}\right\}_{n=1}^{\infty}=\left\{G \gamma_{n}\right\}_{n=1}^{\infty}$ is monotone and bounded and therefore it has a pointwise limit, say $z$.

We are going to prove that $\left\{z_{n}\right\}_{n=1}^{\infty}$ tends to $z$ uniformly on $\left[a-r_{-}, b+r_{+}\right]$and that $z \in \mathcal{Y}$. 
First, for $s, t \in[a, b], s<t$, and every $n \in \mathbb{N}$ we have

$$
\left|z_{n}(t)-z_{n}(s)\right| \leq \int_{s}^{t}\left|z_{n}^{\prime}(r)\right| d r \leq \int_{s}^{t} \rho(r) d r .
$$

Second, for $s, t \in I_{-}$and $n \in \mathbb{N}$ we have

$$
\begin{aligned}
\left|z_{n}(t)-z_{n}(s)\right|= & \left|B_{-}\left(t, z_{n}(t), \gamma_{n}\right)-B_{-}\left(s, z_{n}(s), \gamma_{n}\right)\right| \\
\leq & \left|B_{-}\left(t, z_{n}(t), \gamma_{n}\right)-B_{-}\left(t, z_{n}(s), \gamma_{n}\right)\right| \\
& +\left|B_{-}\left(t, z_{n}(s), \gamma_{n}\right)-B_{-}\left(s, z_{n}(s), \gamma_{n}\right)\right| \\
\leq & \lambda\left|z_{n}(t)-z_{n}(s)\right|+\left|B_{-}\left(t, z_{n}(s), \gamma_{n}\right)-B_{-}\left(s, z_{n}(s), \gamma_{n}\right)\right|,
\end{aligned}
$$

hence

$$
\left|z_{n}(t)-z_{n}(s)\right| \leq \frac{1}{1-\lambda}\left|B_{-}\left(t, z_{n}(s), \gamma_{n}\right)-B_{-}\left(s, z_{n}(s), \gamma_{n}\right)\right| .
$$

Analogously, for $s, t \in I_{+}$and $n \in \mathbb{N}$ we have

$$
\left|z_{n}(t)-z_{n}(s)\right| \leq \frac{1}{1-\lambda}\left|B_{+}\left(t, z_{n}(s), \gamma_{n}\right)-B_{+}\left(s, z_{n}(s), \gamma_{n}\right)\right| .
$$

Since the $z_{n}$ are continuous at $a$ and $b$, we deduce from (2.4), (2.5), (2.6), and condition $\left(\mathrm{B}_{1}\right)$ that $\left\{z_{n}\right\}_{n=1}^{\infty}$ is uniformly equicontinuous on $\left[a-r_{-}, b+r_{+}\right]$. Hence $\left\{z_{n}\right\}_{n=1}^{\infty}$ tends to $z$ uniformly on $\left[a-r_{-}, b+r_{+}\right]$and $z$ is continuous on $\left[a-r_{-}, b+r_{+}\right]$.

Moreover, (2.4) implies that $z$ is absolutely continuous on $[a, b]$, thus proving that $z \in \mathcal{Y}$.

Now we can apply Lemma 2.1 to ensure that operator $G$ has the extremal fixed points in $\left[\sigma_{1}, \sigma_{2}\right]$.

Claim 4: The greatest fixed point of $G$ corresponds with the greatest solution of problem (2.1) in $\left[\sigma_{1}, \sigma_{2}\right]$. Let $u^{*}$ be the greatest fixed point of $G$. As $G u^{*}=u^{*}$, it is clear that $u^{*}$ is a solution of problem (2.1). Now, let $u \in\left[\sigma_{1}, \sigma_{2}\right]$ be another solution of (2.1). In this case, both $u$ and $G u$ solve $\left(P_{u}\right)$, and so $u \leq G u$ taking into account that $G u$ is the greatest of such solutions. Now, condition (2.2) implies that $u \leq u^{*}$.

The existence of the least solution of (2.1) in $\left[\sigma_{1}, \sigma_{2}\right]$ follows from a proper redefinition of the operator $G$.

The next less general version of Theorem 2.1 is easier to use in practice.

Corollary 2.1 Under the conditions of Theorem 2.1, replace $\left(\mathrm{F}_{1}\right)$ by

$\left(\mathrm{F}_{1}\right)^{\prime}$ For each $\gamma \in\left[\sigma_{1}, \sigma_{2}\right]$ the function $f_{\gamma}(t, u, v)=f(t, u, v, \gamma)$ satisfies $\left(\mathrm{H}_{1}\right)-\left(\mathrm{H}_{4}\right)$ with the same function $\rho$ in $\left(\mathrm{H}_{4}\right)$ for all $\gamma \in\left[\sigma_{1}, \sigma_{2}\right]$.

Then the conclusion of Theorem 2.1 holds.

\section{An example of application}

We finish this paper with a corollary of our main result which applies to a large family of boundary conditions satisfying stronger conditions that $\left(B_{1}\right)-\left(B_{3}\right)$. This corollary is illustrated later with a concrete example. 
Corollary 3.1 Let $f$ satisfy $\left(\mathrm{F}_{1}\right)\left(\right.$ or $\left.\left(\mathrm{F}_{1}\right)^{\prime}\right)$ and $\left(\mathrm{F}_{2}\right)$ and assume that the functions $B_{-}, B_{+}$ can be written as follows:

$B_{\mp}(t, u, \gamma)=\psi_{1}(t)+\psi_{2}(t, \gamma) \psi_{3}(u)$.

Then the following conditions are sufficient to guarantee the conclusion of Theorem 2.1:

$\left(\widetilde{\mathrm{B}}_{1}\right)$ The function $\psi_{1}$ is uniformly continuous in $(a, b)$.

$\left(\widetilde{\mathrm{B}}_{2}\right)$ For all $t \in(a, b)$ and all $\gamma \in\left[\sigma_{1}, \sigma_{2}\right]$ the function $\psi_{2}(\cdot, \gamma)$ is Lipschitzian with Lipschitz constant $L_{\gamma}$, and the function $\psi_{2}(t, \cdot)$ is nondecreasing. Moreover, there exists $L=\sup \left\{L_{\gamma}: \gamma \in\left[\sigma_{1}, \sigma_{2}\right]\right\}$.

$\left(\widetilde{\mathrm{B}}_{3}\right)$ For all $u \in\left[\min \sigma_{1}, \max \sigma_{2}\right]$ the function $\psi_{3}$ is nonnegative, differentiable, and

$$
\left|\psi_{3}^{\prime}(u)\right|<\left(\max _{t \in(a, b)} \max \left\{\left|\psi_{2}\left(t, \sigma_{1}\right)\right|,\left|\psi_{2}\left(t, \sigma_{2}\right)\right|\right\}\right)^{-1}
$$

Proof We will show that these conditions imply $\left(\mathrm{B}_{1}\right)-\left(\mathrm{B}_{3}\right)$.

To see $\left(\mathrm{B}_{1}\right)$, we take $t, s \in(a, b)$ and apply $\left(\widetilde{\mathrm{B}}_{2}\right)$ to obtain for $u \in\left[\min \sigma_{1}, \max \sigma_{2}\right]$ and $\gamma \in\left[\sigma_{1}, \sigma_{2}\right]$ that

$$
\left|B_{\mp}(t, u, \gamma)-B_{\mp}(s, u, \gamma)\right| \leq\left|\psi_{1}(t)-\psi_{1}(s)\right|+\psi_{3}(u) L|t-s|,
$$

and now $\left(\mathrm{B}_{1}\right)$ follows from $\left(\widetilde{\mathrm{B}}_{1}\right)$ and the fact that $\psi_{3}$ is bounded in the compact set $\left[\min \sigma_{1}, \max \sigma_{2}\right]$.

To see $\left(\mathrm{B}_{2}\right)$ we notice that for $u, v \in\left[\min \sigma_{1}, \max \sigma_{2}\right], u<v$, condition $\left(\widetilde{\mathrm{B}}_{3}\right)$ provides that there exists $\xi \in(u, v)$ such that

$$
\left|B_{\mp}(t, u, \gamma)-B_{\mp}(t, v, \gamma)\right| \leq\left|\psi_{2}(t, \gamma)\right|\left|\psi_{3}^{\prime}(\xi)\right||u-v| \quad \text { for all } t \in(a, b), \gamma \in\left[\sigma_{1}, \sigma_{2}\right] .
$$

Then $\left(\mathrm{B}_{2}\right)$ follows from (3.1).

Finally, condition $\left(\widetilde{\mathrm{B}}_{3}\right)$ follows from the facts that $\psi_{2}(t, \cdot)$ is nondecreasing and $\psi_{3}$ is nonnegative.

Example 3.1 Let $\phi_{i}:[0,1] \rightarrow \mathbb{R}, i=1,2$, be nonincreasing functions (not necessarily continuous), and consider the following functional problem, which includes both a past and a future dependence:

$$
\left\{\begin{array}{l}
u^{\prime \prime}(t)=\frac{u^{2}(t)\left|u^{\prime}(t)\right|}{\lambda t(1-t)}\left[\phi_{1}(u(t-1 / 2))+\phi_{2}(u(t+1 / 2))\right] \quad \text { for a.a. } t \in(0,1), \\
u(t)=\int_{-1 / 2}^{t+1 / 2} k(s) u(s) d s \quad \text { for all } t \in[-1 / 2,0] \\
(2-u(t))^{2 / 3}=\sqrt{\left|\frac{5}{2}-t \max _{t \in[-1 / 2,3 / 2]} u(t)\right|} \text { for all } t \in[1,3 / 2]
\end{array}\right.
$$

where $k \in L^{1}([-1 / 2,1 / 2],[0,+\infty))$.

We claim that for sufficiently small value of $\|k\|_{1}$ problem (3.2) has the extremal solutions between the lower solution $\sigma_{1} \equiv 0$ and the upper solution $\sigma_{2} \equiv 1$. Notice that $\sigma_{1} \equiv 0$ and $\sigma_{2} \equiv 1$ are solutions of the differential equation in (3.2) but not solutions of the whole problem. 
This problem falls inside the scope of our main result. To see it, simply define the functions

$$
\begin{aligned}
& f(t, u, v, \gamma)=\frac{u^{2}|v|}{\lambda t(1-t)}\left[\phi_{1}(\gamma(t-1 / 2))+\phi_{2}(\gamma(t+1 / 2))\right], \\
& B_{-}(t, \gamma)=\psi_{2-}(t, \gamma) \\
& B_{+}(t, u, \gamma)=2+\psi_{2+}(t, \gamma) \psi_{3+}(u),
\end{aligned}
$$

where

$$
\psi_{2-}(t, \gamma)=\int_{-1 / 2}^{t+1 / 2} k(s) \gamma(s) d s
$$

and

$$
\psi_{2+}(t, \gamma)=-\sqrt{\left|\frac{5}{2}-t \max _{t \in[-1 / 2,3 / 2]} \gamma(t)\right|}, \quad \psi_{3+}(u)=(2-u)^{1 / 3} .
$$

We are going to use Corollary 3.1 to prove that problem (3.2) has the extremal solutions between $\sigma_{1} \equiv 0$ and $\sigma_{2} \equiv 1$ provided that

$$
\frac{1}{\tilde{\lambda}}=\frac{\max \left\{\left|\phi_{1}(0)+\phi_{2}(0)\right|,\left|\phi_{1}(1)+\phi_{2}(1)\right|\right\}}{\lambda} \in(0,1)
$$

and

$$
\int_{-1 / 2}^{1 / 2} k(s) d s \leq 1
$$

First we have to prove that $\sigma_{1}$ and $\sigma_{2}$ are, in fact, lower and upper solutions. To see that $\sigma_{1}$ is a lower solution, notice that $B_{-}(t, 0)=0$ and $B_{+}(t, 0,0)=2-2^{1 / 3} \sqrt{5 / 2} \geq 0$. Now, to check that $\sigma_{2}$ is an upper solution, we use

$$
B_{-}(t, 1)=\int_{-1 / 2}^{t+1 / 2} k(s) d s \leq 1 \quad \text { by virtue of (3.4) }
$$

and

$$
B_{+}(t, 1,1)=2-\sqrt{|5 / 2-t|} \leq 1 \quad \text { for } t \in(1,3 / 2) .
$$

Let us check the assumptions required in Corollary 3.1. For all $(t, u, v, \gamma) \in(0,1) \times[0,1] \times$ $\mathbb{R} \times\left[\sigma_{1}, \sigma_{2}\right]$ we have

$$
|f(t, u, v, \gamma)| \leq \frac{u^{2}|v|}{\lambda t(1-t)}\left|\phi_{1}\left(\gamma\left(t-r_{-}\right)\right)+\phi_{2}\left(\gamma\left(t+r_{+}\right)\right)\right| \leq \frac{|v|+1}{\tilde{\lambda} t(1-t)}
$$

and then the condition $\left(\mathrm{H}_{4}\right)$ is satisfied as in Example 1.1 (with the same function $\rho$ for all $\left.\gamma \in\left[\sigma_{1}, \sigma_{2}\right]\right)$, thanks to condition (3.3). 
The remaining conditions over $f$ are easily verified, and therefore we omit further explanations.

Now for the boundary conditions. First, both $\psi_{2-}$ and $\psi_{2+}$ are bounded w.r.t. $t$ and nondecreasing w.r.t. $\gamma$, and for $s, t \in[-1 / 2,0]$ and $\gamma \in\left[\sigma_{1}, \sigma_{2}\right]$ we have

$$
\left|\psi_{2-}(t, \gamma)-\psi_{2+}(s, \gamma)\right| \leq\left|\int_{s+1 / 2}^{t+1 / 2} k(s) d s\right| \leq|t-s|\|k\|_{1},
$$

so $\psi_{2-}(\cdot, \gamma)$ is Lipschitzian for all $\gamma$ with Lipschtitz constant $\|k\|_{1}$. On the other hand, the function $\psi_{2+}$ satisfies

$$
\left|\frac{\partial}{\partial t} \psi_{2+}(t, \gamma)\right| \leq \frac{1}{2}
$$

and so condition $\left(\widetilde{\mathrm{B}}_{2}\right)$ holds.

Finally, the function $\psi_{3_{+}}(u)=(2-u)^{1 / 3}$ is nonnegative for $u \in[0,1]$ and satisfies

$$
\left|\psi_{3+}^{\prime}(u)\right| \leq \frac{1}{3} \leq \sqrt{\frac{2}{5}}=\left|\psi_{2+}(t, 0)\right|^{-1} \quad \text { for all } t \in(a, b),
$$

and so condition $\left(\widetilde{\mathrm{B}}_{3}\right)$ holds.

\section{Competing interests}

The authors declare they have no competing interest.

\section{Authors' contributions}

The authors worked jointly on this paper and their respective contributions were of similar quality and quantity. All authors read and approved the final manuscript.

\section{Author details}

${ }^{1}$ Department of Specific Didactics, University of Burgos, Burgos, 09001, Spain. ${ }^{2}$ Department of Mathematical Analysis, University of Santiago de Compostela, Santiago de Compostela, 15782, Spain.

\section{Acknowledgements}

This work was partially supported by FEDER and Ministerio de Educación y Ciencia, Spain, project MTM2010-15314.

Received: 13 December 2013 Accepted: 13 June 2014 Published online: 24 September 2014

\section{References}

1. Kiguradze, IT, Partsvania, N: On minimal and maximal solutions of two-point singular boundary value problems. Mem. Differ. Equ. Math. Phys. 36, 147-152 (2005)

2. Kiguradze, IT: On some singular boundary value problems for nonlinear second order ordinary differential equations. Differ. Uravn. 4(10), 1753-1773 (1968) (Russian); Differ. Equ. 4, 901-910 (1968) (English translation)

3. Kiguradze, IT, Staněk, S: On periodic boundary value problem for the equation $u^{\prime \prime}=f\left(t, u, u^{\prime}\right)$ with one-sided growth restrictions on $f$. Nonlinear Anal. 48, 1065-1075 (2002)

4. Adje, A: Sur et sous-solutions généralisées et problèmes aux limites du second ordre. Bull. Soc. Math. Belg., Sér. B 42, 347-368 (1990)

5. De Coster, C, Habets, P: Two-Point Boundary Value Problems: Lower and Upper Solutions. Mathematics in Science and Engineering, vol. 205. Elsevier, Amsterdam (2006)

6. López Pouso, R: Some general comparison results for second order ordinary differential equations. J. Math. Anal. Appl. $252,710-728(2000)$

7. Cabada, A, O'Regan, D, Pouso, RL: Second order problems with functional conditions including Sturm-Liouville and multipoint conditions. Math. Nachr. 281, 1254-1263 (2008)

8. Kiguradze, IT, Lomtatidze, A, Partsvania, N: Some multi-point boundary value problems for second order singular differential equations. Mem. Differ. Equ. Math. Phys. 56, 133-141 (2012)

9. Heikkilä, S, Lakshmikantham, V: Monotone Iterative Techniques for Discontinuous Nonlinear Differential Equations. Dekker, New York (1994) 